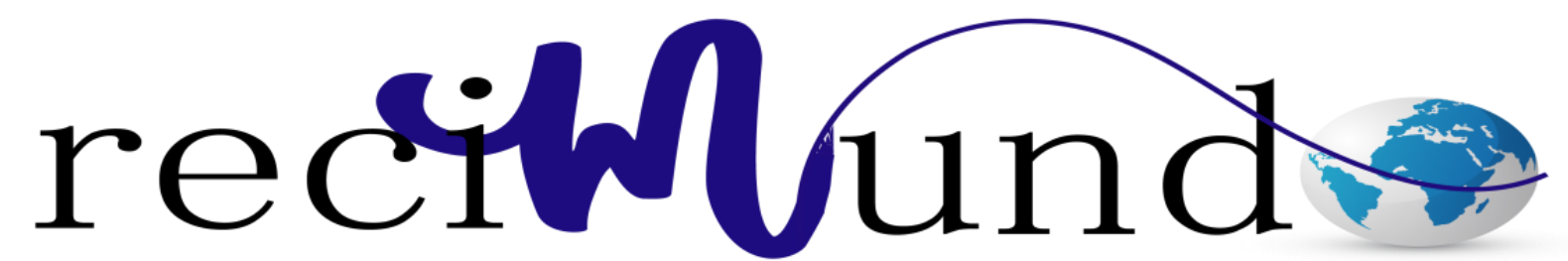

Revista Cientifica Mundo de la Investigación y el Conocímiento

\author{
Maria Monserrate Ganchozo Pincay a; Ginger Alexandra Macías Corral ${ }^{\text {b; }}$ Marlon \\ Jose Rivadeneira Rodriguez ${ }^{\mathrm{c}}$; José Andrés Sánchez Acebo ${ }^{\mathrm{d}}$
}

Fisiopatología de los problemas respiratorios en pacientes asmáticos

Pathophysiology of respiratory problems in asthmatic patients

Revista Científica Mundo de la Investigación y el Conocimiento. Vol. 3 núm.3. Esp., noviembre, ISSN: 2588-073X, 2019, pp. 942-961

DOI: $10.26820 /$ recimundo/3.(3.Esp).noviembre.2019.942-961

URL: http://recimundo.com/index.php/es/article/view/635

Código UNESCO: 3205 Medicina Interna

Tipo de Investigación: Artículo de Revisión

(C) RECIMUNDO; Editorial Saberes del Conocimiento, 2019

Recibido: 15/09/2019

Aceptado: 23/10/2019

Publicado: 30/11/2019

Correspondencia: gp_mayte@hotmail.com

a. Médico; Investigadora Independiente; Guayaquil, Ecuador; gp_mayte@ hotmail.com

b. Médico; Investigadora Independiente; Guayaquil, Ecuador; ginger.ale.mac@ gmail.com

c. Médico; Investigador Independiente; Guayaquil, Ecuador; marlon.riro94@ gmail.com

d. Médico; Investigador Independiente; Guayaquil, Ecuador; $\underline{\text { andresancheza@ hotmail.com }}$ 


\section{Fisiopatología de los problemas respiratorios en pacientes asmáticos}

Vol. 3, núm. 3 Esp., (2019)

Maria Monserrate Ganchozo Pincay; Ginger Alexandra Macías Corral; Marlon Jose Rivadeneira

Rodriguez; José Andrés Sánchez Acebo

\section{RESUMEN}

El asma es una enfermedad crónica de las vías aéreas que afecta a más de 300 millones de personas en todo el mundo, generando aproximadamente 250,000 muertes anuales. A medida que se conocen los mecanismos biológicos de esta patología y los diferentes fenotipos moleculares que participan en ella, se ha podido cambiar, si bien de manera lenta, su enfoque diagnóstico y terapéutico, y han comenzado a vislumbrarse alternativas farmacológicas que reforzarán el armamento terapéutico, hasta ahora disponible para tratarla. La metodología empleada en la investigación es de tipo revisión bibliográfica apoyada en medios electrónicos. Entre las conclusiones más relevantes se tienen Las pruebas de función pulmonar son de gran utilidad en el diagnóstico, evaluación de la gravedad de la obstrucción y en el seguimiento de la respuesta al tratamiento. Las pruebas de alergia son necesarias, principalmente para establecer si la exposición a alérgenos influye en el desarrollo del asma y con ello diseñar las medidas de control necesarias para evitar la exposición a alérgenos y la aparición de síntomas. El asmático es un paciente crónico difícil de manejar, que requiere de una terapia compleja; su manejo debe ser integral, y un punto básico es la educación del paciente y su familia.

Palabras clave: Asma; Fisiopatología; Vías; Aéreas; Alérgenos. 
Fisiopatología de los problemas respiratorios en pacientes asmáticos

Vol. 3, núm. 3 Esp., (2019)

Maria Monserrate Ganchozo Pincay; Ginger Alexandra Macías Corral; Marlon Jose Rivadeneira Rodriguez; José Andrés Sánchez Acebo

\begin{abstract}
Asthma is a chronic disease of the airways that affects more than 300 million people worldwide, generating approximately 250,000 deaths annually. As the biological mechanisms of this pathology and the different molecular phenotypes that participate in it are known, its diagnostic and therapeutic approach has been changed, albeit slowly, and pharmacological alternatives that will strengthen the therapeutic armament have begun to be envisioned., so far available to treat it. The methodology used in the research is of a bibliographic review type based on electronic media. Among the most relevant conclusions are Pulmonary function tests are very useful in the diagnosis, evaluation of the severity of the obstruction and in monitoring the response to treatment. Allergy tests are necessary, mainly to establish if allergen exposure influences the development of asthma and thereby design the necessary control measures to avoid allergen exposure and the appearance of symptoms. The asthmatic is a chronic patient difficult to manage, requiring complex therapy; its management must be integral, and a basic point is the education of the patient and his family.
\end{abstract}

Keywords: Asthma; Pathophysiology; Roads; Aerial; Allergens. 


\section{Fisiopatología de los problemas respiratorios en pacientes asmáticos}

Vol. 3, núm. 3 Esp., (2019)

Maria Monserrate Ganchozo Pincay; Ginger Alexandra Macías Corral; Marlon Jose Rivadeneira

Rodriguez; José Andrés Sánchez Acebo

\section{Introducción.}

El asma se presenta cada vez más, como una situación clínica de heterogenia y complejidad extremas. A medida que se conocen los mecanismos biológicos de esta patología y los diferentes fenotipos moleculares que participan en ella, se ha podido cambiar, si bien de manera lenta, su enfoque diagnóstico y terapéutico, y han comenzado a vislumbrarse alternativas farmacológicas que reforzarán el armamento terapéutico, hasta ahora disponible para tratarla (Durán, 2015, pág. 226).

El asma es una enfermedad crónica de las vías aéreas que afecta a más de 300 millones de personas en todo el mundo, generando aproximadamente 250,000 muertes anuales. El asma alérgica es el fenotipo clínico más común de la enfermedad. En su etiología influyen múltiples factores genéticos y medioambientales y en su desarrollo participan muchas células inflamatorias como mastocitos, eosinófilos, neutrófilos, linfocitos $\mathrm{T}$ y células epiteliales. Los mediadores inflamatorios liberados por estas células generan y mantienen un estado de inflamación crónica que induce broncoconstricción, hiperreactividad bronquial y cambios estructurales en las vías aéreas. Descifrar y entender los mecanismos inmunológicos exactos involucrados en el desarrollo de asma alérgica es de crucial importancia para el desarrollo de nuevos tratamientos. El propósito de este trabajo es revisar la información más reciente sobre los elementos moleculares y celulares relacionados con el asma alérgica, así como su fisiopatología y los tratamientos actuales (Bretón, Vargas, \& Miralles, 2015, pág. 66).

La inflamación y remodelación de la vía aérea son dos características del asma que se han estudiado profundamente en los últimos 20 años; el reconocimiento de que la inflamación es el 


\section{Fisiopatología de los problemas respiratorios en pacientes asmáticos}

Vol. 3, núm. 3 Esp., (2019)

Maria Monserrate Ganchozo Pincay; Ginger Alexandra Macías Corral; Marlon Jose Rivadeneira Rodriguez; José Andrés Sánchez Acebo

hecho clave en el asma, producida por una compleja interacción entre células inflamatorias y células residentes de la vía aérea, ha llevado a formular hipótesis sobre cuál o cuáles de éstas células puedan dirigir este proceso. Así mastocitos, eosinófilos, células epiteliales y linfocitos CD4 han sido propuestos cómo las células que conducen el proceso inflamatorio. Existe un creciente consenso sobre la teoría de la evolución del asma, que propone que individuos susceptibles genéticamente y que están expuestos tempranamente en la vida a un medio ambiente específico, desarrollan un tipo peculiar de inflamación linfocítica de la vía aérea que resulta en asma (González \& Pérez, 2006, pág. 49).

Figura I.- Orígenes y evolución del asma según S. Holgate

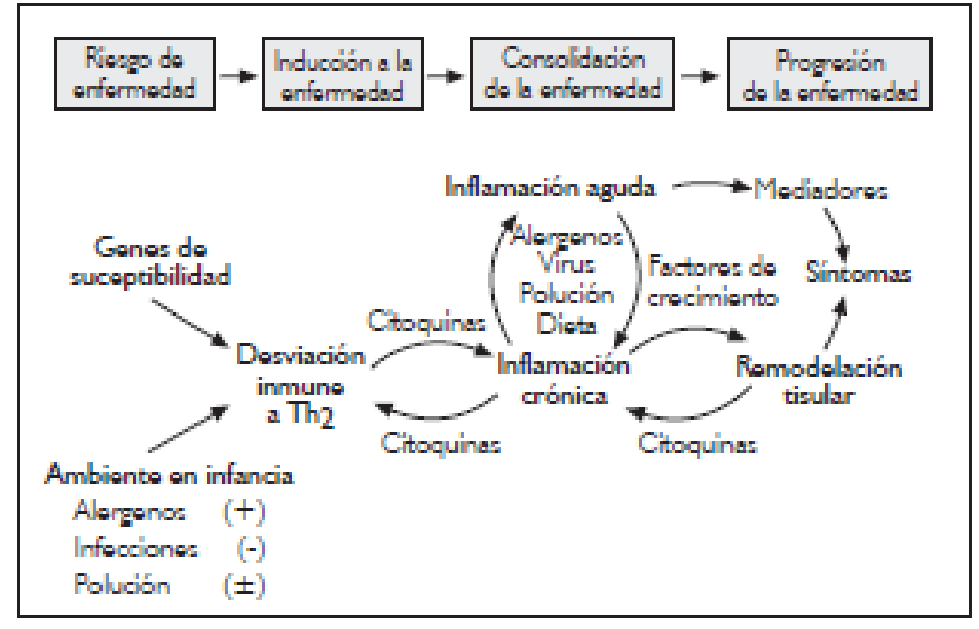

Fuente: (González \& Pérez, 2006).

Podemos dividir la respuesta asmática en dos: la inmediata (RI) y la tardía (RT). La primera es de inicio rápido, se presenta minutos después de la exposición al desencadenante y alcanza su pico máximo a los 15 minutos, con una duración de 1 a 2 horas; progresa rápidamente y se presenta en $35 \%$ de los casos. Su característica principal es que depende del espasmo del 


\section{Fisiopatología de los problemas respiratorios en pacientes asmáticos}

Vol. 3, núm. 3 Esp., (2019)

Maria Monserrate Ganchozo Pincay; Ginger Alexandra Macías Corral; Marlon Jose Rivadeneira Rodriguez; José Andrés Sánchez Acebo

músculo liso bronquial y afecta las vías aéreas de grueso calibre. La RT, por su parte, inicia de 2 a 4 horas después del estímulo; su máxima acción se presenta entre 5 y 12 horas después, y puede llegar a durar varios días; progresa lenta e intensamente y afecta a las vías de pequeño calibre; se presenta en $25 \%$ de los pacientes. Los broncodilatadores tienen poco efecto, por lo que se considera que la broncoconstricción, en esta etapa, no es tan importante como la inflamación (Sienra-Monge, Río-Navarro, \& Baeza-Bacab, M, 1999, pág. 65).

Durante la espiración, el volumen de la caja torácica tiende a disminuir; en una espiración tranquila normal, el diafragma y los músculos inspiratorios se relajan y la elasticidad de la caja torácica tiende a regresar a su posición de reposo (FRC). Esto provoca compresión pasiva de los pulmones, que es favorecida por la propia retracción elástica pulmonar. Sin embargo, el flujo aéreo nuevamente debe vencer la resistencia de la vía aérea pero ahora la resistencia es mayor, ya que la compresión pulmonar disminuye el calibre de las vías aéreas e incluso, cuando el volumen pulmonar es muy bajo, tiende a cerrar por completo la vía aérea periférica, limitando el flujo espiratorio (Becerra, 2009, pág. 112). 


\section{Fisiopatología de los problemas respiratorios en pacientes asmáticos}

Vol. 3, núm. 3 Esp., (2019)

Maria Monserrate Ganchozo Pincay; Ginger Alexandra Macías Corral; Marlon Jose Rivadeneira Rodriguez; José Andrés Sánchez Acebo

Figura 2. Representación esquemática de los cambios en la presión transpulmonar y el calibre de las vías aéreas durante el ciclo respiratorio.

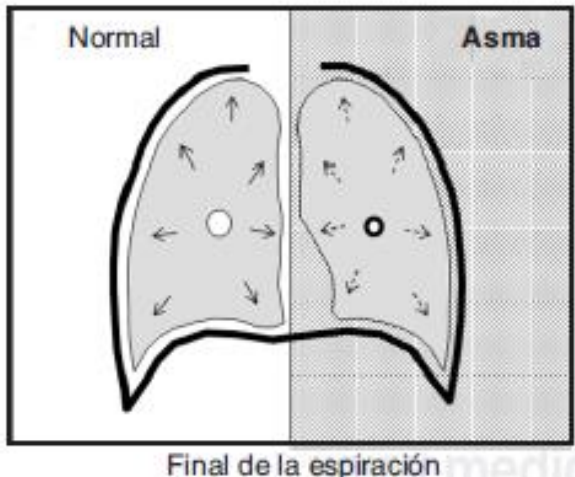

Final de la espiración

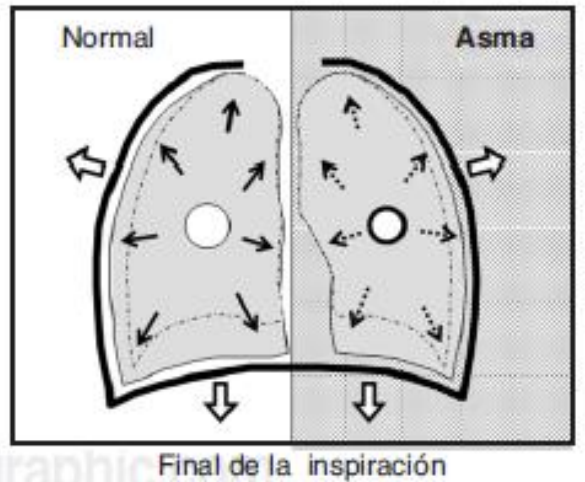

Final de la inspiración

Fuente: (Becerra, 2009).

En condiciones normales, al final de una espiración tranquila (panel izquierdo) hay un equilibrio entre la presión transpulmonar (PL, flechas continuas) y la caja torácica. Esta presión se transmite por las paredes alveolares hasta las vías aéreas (círculo), favoreciendo su apertura. Durante la inspiración (panel derecho) la expansión pulmonar hace que se intensifique la PL, lo que aumenta el diámetro de las vías aéreas. En el paciente asmático hay una disminución de la PL (flechas discontinuas), por lo que las vías aéreas no son jaladas tan eficazmente y tienden a estar más cerradas en todo momento, o incluso a colapsarse prematuramente al final de la espiración (Becerra, 2009, pág. 112).

\section{Metodología.}

La metodología empleada en la presente investigación es de tipo revisión bibliográfica, para lo cual se han utilizado diferentes medios electrónicos, donde se han encontrado una amplia gama de trabajos de investigación que abordan el tema de la fisiopatología del asma, para lo cual 
Fisiopatología de los problemas respiratorios en pacientes asmáticos

Vol. 3, núm. 3 Esp., (2019)

Maria Monserrate Ganchozo Pincay; Ginger Alexandra Macías Corral; Marlon Jose Rivadeneira Rodriguez; José Andrés Sánchez Acebo

se han escogidos los de mayor relevancia para presentar un compendio de resultados y conclusiones a esta patología que afecta a cientos de millones de personas a nivel mundial.

\section{Resultados.}

Tabla 1. Pruebas de función pulmonar

\begin{tabular}{|l|l|}
\hline Reversibilidad del flujo espiratorio & $\begin{array}{l}\text { La reversibilidad se determina, primero, } \\
\text { midiendo el PEF con el flujómetro; } \\
\text { posteriormente se administra al paciente } \\
\text { un broncodilatador y se esperan de 15 a } 20 \\
\text { minutos; una vez transcurrido este tiempo } \\
\text { se repite la medición del PEF; un cambio } \\
\text { mayor al 20\% respecto del valor inicial } \\
\text { (antes de administrar el broncodilatador) } \\
\text { es sugestivo de asma. Antes de realizar } \\
\text { esta prueba se le debe indicar al paciente } \\
\text { no utilizar ningún broncodilatador. }\end{array}$ \\
\hline Para obtener la variabilidad, se le pide al \\
paciente que lleve un registro del PEF \\
durante dos semanas o más. El paciente \\
debe medir el PEF por la mañana y por la
\end{tabular}


Fisiopatología de los problemas respiratorios en pacientes asmáticos

Vol. 3, núm. 3 Esp., (2019)

Maria Monserrate Ganchozo Pincay; Ginger Alexandra Macías Corral; Marlon Jose Rivadeneira Rodriguez; José Andrés Sánchez Acebo

\begin{tabular}{|c|c|}
\hline $\begin{array}{c}\text { Variabilidad del flujo espiratorio } \\
\text { máximo }\end{array}$ & $\begin{array}{l}\text { tarde o noche, haciendo tres mediciones y } \\
\text { registrando el valor más alto de éstas; el } \\
\text { valor de las mediciones debe ser muy } \\
\text { parecido entre sí. Una variabilidad > del } \\
20 \% \text {, sugiere el diagnóstico de asma }\end{array}$ \\
\hline \multirow[t]{2}{*}{ Prueba de reto con metacolina } & $\begin{array}{l}\text { Esta prueba mide el grado de } \\
\text { hiperreactividad bronquial; las sustancias } \\
\text { más utilizadas son la metacolina y la } \\
\text { histamina por vía inhalada y consiste en } \\
\text { que el sujeto inhale dosis crecientes de } \\
\text { estas sustancias hasta que el FEV1 } \\
\text { disminuya } 20 \% \text { respecto al basal (PC20). } \\
\text { La prueba de reto negativa descarta el } \\
\text { asma en un paciente con síntomas y } \\
\text { también es útil en el diagnóstico y } \\
\text { seguimiento del asma laboral. En caso de } \\
\text { sospecha de asma inducida por ejercicio, } \\
\text { se puede realizar una prueba de reto con } \\
\text { ejercicio. }\end{array}$ \\
\hline & Esta prueba es particularmente útil en \\
\hline
\end{tabular}


Fisiopatología de los problemas respiratorios en pacientes asmáticos

Vol. 3, núm. 3 Esp., (2019)

Maria Monserrate Ganchozo Pincay; Ginger Alexandra Macías Corral; Marlon Jose Rivadeneira

Rodriguez; José Andrés Sánchez Acebo

\begin{tabular}{|l|l|}
\hline Prueba de reto con ejercicio & \begin{tabular}{l} 
niños, o adultos jóvenes que tienen una \\
historia muy sugestiva de asma cuyas \\
flujometrías son normales y no tienen \\
reversibilidad con el broncodilatador. Esta \\
prueba se basa en el broncoespasmo \\
inducido por el ejercicio \\
\hline Medición de óxido nítrico
\end{tabular} \mid $\begin{array}{l}\text { La medición de la fracción de óxido } \\
\text { nítrico exhalado es útil en el diagnóstico } \\
\text { de asma, determina en forma no invasiva } \\
\text { la inflamación eosinofílica de la vía aérea, } \\
\text { y es de gran utilidad en el seguimiento de } \\
\text { los pacientes bajo tratamiento con } \\
\text { antiinflamatorios esteroideos. Tiene alta } \\
\text { sensibilidad para medir la inflamación aun } \\
\text { en pacientes asintomáticos, para valorar } \\
\text { los cambios en el tratamiento } \\
\text { y puede ser un predictor de las } \\
\text { exacerbaciones. Es un procedimiento } \\
\text { dependiente de factores técnicos y } \\
\text { metodológicos; en los centros de atención } \\
\text { donde el procedimiento está estandarizado }\end{array}$ \\
\hline
\end{tabular}


Fisiopatología de los problemas respiratorios en pacientes asmáticos

Vol. 3, núm. 3 Esp., (2019)

Maria Monserrate Ganchozo Pincay; Ginger Alexandra Macías Corral; Marlon Jose Rivadeneira Rodriguez; José Andrés Sánchez Acebo

\begin{tabular}{|c|c|}
\hline & $\begin{array}{l}\text { el valor de } 20 \text { a } 30 \text { ppb (partes por billón) } \\
\text { es diagnóstico de asma. }\end{array}$ \\
\hline $\begin{array}{c}\text { Medición de células inflamatorias en } \\
\text { expectoración }\end{array}$ & $\begin{array}{l}\text { Esta prueba es útil no sólo para el } \\
\text { diagnóstico de asma, sino también para la } \\
\text { diferenciación } \\
\text { de algunos tipos de asma; por ejemplo. en } \\
\text { el } \\
\text { asma de difícil control se pueden } \\
\text { encontrar predominantemente neutrófilos } \\
\text { a diferencia del asma alérgica en la cual } \\
\text { son los eosinófilos las células } \\
\text { predominantes }\end{array}$ \\
\hline Pruebas alérgicas & $\begin{array}{l}\text { Las pruebas de alergia proporcionan } \\
\text { información que puede ayudar a } \\
\text { establecer si la exposición a alergenos } \\
\text { influye en el desarrollo del asma, además } \\
\text { son útiles para crear medidas que } \\
\text { controlen la exposición a alergenos y la } \\
\text { aparición de síntomas. Son pruebas que }\end{array}$ \\
\hline
\end{tabular}


Fisiopatología de los problemas respiratorios en pacientes asmáticos

Vol. 3, núm. 3 Esp., (2019)

Maria Monserrate Ganchozo Pincay; Ginger Alexandra Macías Corral; Marlon Jose Rivadeneira

Rodriguez; José Andrés Sánchez Acebo

\begin{tabular}{|l|l|}
\hline & $\begin{array}{l}\text { debe realizar el alergólogo y el mismo } \\
\text { debe indicar el tratamiento en caso de } \\
\text { resultar positivas. }\end{array}$ \\
\hline
\end{tabular}

Fuente: (García Merino \& Mora Gandarillas, 2013). Elaboración Propia.

Tabla 2. Factores dependientes del huésped

\begin{tabular}{|l|l|}
\hline Genéticos & $\begin{array}{l}\text { El asma tiene un componente hereditario, } \\
\text { pero no es simple. Los datos actuales } \\
\text { muestran que múltiples genes pueden } \\
\text { encontrarse implicados en la patogénesis } \\
\text { del asma, y diferentes genes pueden estar } \\
\text { implicados en distintos grupos étnicos. } \\
\text { Los genes que predisponen al asma son } \\
\text { genes que también se han asociado con la } \\
\text { respuesta al tratamiento del asma; por } \\
\text { ejemplo, variaciones en el gen codificador } \\
\text { del receptor beta adrenérgico han sido } \\
\text { vinculados a diferencias en la respuesta de } \\
\text { los beta agonistas. }\end{array}$ \\
\hline
\end{tabular}


Fisiopatología de los problemas respiratorios en pacientes asmáticos

Vol. 3, núm. 3 Esp., (2019)

Maria Monserrate Ganchozo Pincay; Ginger Alexandra Macías Corral; Marlon Jose Rivadeneira Rodriguez; José Andrés Sánchez Acebo

\begin{tabular}{|c|c|}
\hline Obesidad & $\begin{array}{l}\text { Se ha demostrado la obesidad como un } \\
\text { factor de riesgo de asma. Ciertos } \\
\text { mediadores, tales como la leptina, pueden } \\
\text { afectar a la función de la vía aérea e } \\
\text { incrementar la probabilidad del desarrollo } \\
\text { del asma. }\end{array}$ \\
\hline Sexo & $\begin{array}{l}\text { El sexo masculino es un factor de riesgo } \\
\text { para desarrollar asma en el niño. Antes de } \\
\text { los } 14 \text { años, la prevalencia de asma es, } \\
\text { aproximadamente, dos veces mayor en } \\
\text { niños que en niñas. Durante la } \\
\text { adolescencia, esta diferencia se iguala y, } \\
\text { en el periodo adulto, el asma es más } \\
\text { frecuente en la mujer. }\end{array}$ \\
\hline
\end{tabular}

Fuente: (Casanueva \& Belinchón, 2012). Elaboración Propia.

Tabla 3. Factores desencadenantes ambientales 
Fisiopatología de los problemas respiratorios en pacientes asmáticos

Vol. 3, núm. 3 Esp., (2019)

Maria Monserrate Ganchozo Pincay; Ginger Alexandra Macías Corral; Marlon Jose Rivadeneira

Rodriguez; José Andrés Sánchez Acebo

\begin{tabular}{|l|l|}
\hline A pesar de que los alérgenos, tanto de \\
interior como de exterior, son conocidos \\
como causa de exacerbación asmática, su \\
papel específico en el desarrollo del asma \\
no está enteramente resuelto. Para algunos \\
alérgenos, tales como los derivados del \\
polvo de la casa y de las cucarachas, la \\
prevalencia de sensibilización parece estar \\
correlacionada directamente con la \\
exposición. No obstante, la exposición a \\
los alérgenos de ácaros del polvo puede \\
ser un factor causante del desarrollo del \\
asma. La infestación por cucarachas \\
parece ser un factor importante de causa \\
de sensibilización alérgica en núcleos \\
urbanos. \\
\hline $\begin{array}{l}\text { Ciertas infecciones respiratorias } \\
\text { tempranas en la vida, incluidos el } \\
\text { sarampión y en algún momento el VRS, } \\
\text { pueden proteger del desarrollo de asma. } \\
\text { Respecto a las infecciones parasitarias, }\end{array}$ \\
\hline anfeciones
\end{tabular}


Fisiopatología de los problemas respiratorios en pacientes asmáticos

Vol. 3, núm. 3 Esp., (2019)

Maria Monserrate Ganchozo Pincay; Ginger Alexandra Macías Corral; Marlon Jose Rivadeneira Rodriguez; José Andrés Sánchez Acebo

\begin{tabular}{|c|c|}
\hline & $\begin{array}{l}\text { aunque en general no protegen contra el } \\
\text { asma, la infección por anquilostoma puede } \\
\text { reducir el riesgo }\end{array}$ \\
\hline Exposición al tabaco & $\begin{array}{l}\text { Tanto a nivel prenatal, como postnatal, la } \\
\text { exposición al tabaco se asocia con efectos } \\
\text { perjudiciales, incluido un mayor riesgo de } \\
\text { desarrollar síntomas semejantes al asma } \\
\text { en niños pequeños. No obstante, la } \\
\text { evidencia sobre el aumento del riesgo de } \\
\text { enfermedades alérgicas es incierta. }\end{array}$ \\
\hline \multirow[t]{2}{*}{ Polución } & $\begin{array}{l}\text { La polución ambiental como causa de } \\
\text { asma sigue siendo controvertida. Niños } \\
\text { que viven en un ambiente de alta polución } \\
\text { tienen disminuida su función pulmonar. } \\
\text { Pero la relación entre su baja función } \\
\text { pulmonar y el desarrollo de asma es } \\
\text { desconocida. }\end{array}$ \\
\hline & $\begin{array}{l}\text { Algún dato sugiere que ciertas } \\
\text { características }\end{array}$ \\
\hline
\end{tabular}


Fisiopatología de los problemas respiratorios en pacientes asmáticos

Vol. 3, núm. 3 Esp., (2019)

Maria Monserrate Ganchozo Pincay; Ginger Alexandra Macías Corral; Marlon Jose Rivadeneira

Rodriguez; José Andrés Sánchez Acebo

\begin{tabular}{|l|l|}
\hline de la dieta occidental, tales como: el \\
aumento de consumo de alimentos \\
procesados, la disminución del consumo \\
de antioxidantes (en forma de frutas y \\
verduras), el incremento de \\
ácidos grasos omega-6 poliinsaturados \\
(que se encuentran en la margarina y el \\
aceite vegetal) y la disminución de ácidos \\
grasos omega-3 poliinsaturados (presentes \\
en el pescado) de la ingesta ha contribuido \\
al reciente aumento de asma y \\
enfermedades atópicas. \\
\hline Clima
\end{tabular}


Fisiopatología de los problemas respiratorios en pacientes asmáticos

Vol. 3, núm. 3 Esp., (2019)

Maria Monserrate Ganchozo Pincay; Ginger Alexandra Macías Corral; Marlon Jose Rivadeneira Rodriguez; José Andrés Sánchez Acebo

\begin{tabular}{|l|l|}
\hline & $\begin{array}{l}\text { Por tanto, las personas afectadas de } \\
\text { alergia al polen deberían tener cuidado si } \\
\text { se encuentran en la intemperie durante una } \\
\text { tormenta en la estación polínica. }\end{array}$ \\
\hline
\end{tabular}

Fuente: (Casanueva \& Belinchón, 2012). Elaboración Propia.

El diagnóstico es clínico y, en ocasiones, puede resultar bastante claro; sin embargo, sabemos que en general el asma se subdiagnostica y que frecuentemente se utilizan nombres distintos para referirse a la misma enfermedad. La sintomatología guarda una estrecha relación con los cambios fisiopatológicos; así, el padecimiento presenta tres síntomas cardinales: tos, sibilancias y falta de aire, los cuales no necesariamente tienen que manifestarse juntos, por lo que la ausencia de alguno de ellos no invalida el diagnóstico. La tos es por la estimulación de sus receptores en la vía aérea que se activan al detectar alguna obstrucción al paso del aire. Las sibilancias están determinadas por el paso del aire a través de los bronquios, cuyo calibre se ve disminuido, ya sea por el espasmo del músculo liso, el aumento de secreciones o por el proceso inflamatorio. Por lo que respecta a la falta de aire, se presenta una disminución en la capacidad espiratoria, ya que la obstrucción provoca un retraso en la expulsión del aire; ello a su vez altera el mecanismo de la respiración (Sienra-Monge, Río-Navarro, \& Baeza-Bacab, M, 1999, pág. 66). 


\section{Fisiopatología de los problemas respiratorios en pacientes asmáticos}

Vol. 3, núm. 3 Esp., (2019)

Maria Monserrate Ganchozo Pincay; Ginger Alexandra Macías Corral; Marlon Jose Rivadeneira Rodriguez; José Andrés Sánchez Acebo

Imagen 3. Fenotipos de asma

\begin{tabular}{|c|c|c|c|c|}
\hline & Historia natural & $\begin{array}{l}\text { Características } \\
\text { clínicas y fisiológicas }\end{array}$ & $\begin{array}{l}\text { Biomarcadores y } \\
\text { fisiopatología }\end{array}$ & Respuesta a terapia \\
\hline \multicolumn{5}{|l|}{ Fenotipo TH2-alto } \\
\hline $\begin{array}{l}\text { Asma alérgica } \\
\text { de inicio } \\
\text { temprano }\end{array}$ & $\begin{array}{l}\text { Inicia en infancia, } \\
\text { leve a grave }\end{array}$ & $\begin{array}{l}\text { Síntomas alérgicos } \\
\text { y presenta otras } \\
\text { enfermedades } \\
\text { alérgicas }\end{array}$ & $\begin{array}{c}\text { Engrosamiento } \\
\text { de la membrana } \\
\text { basamental } \\
\text { subepitelial, IgE } \\
\text { especifica, citocinas } \\
\text { TH2 }\end{array}$ & Corticosteroides \\
\hline $\begin{array}{l}\text { Asma } \\
\text { eosinofílica } \\
\text { persistente de } \\
\text { inicio tardío }\end{array}$ & $\begin{array}{c}\text { Inicia en edad adulta, } \\
\text { frecuente- } \\
\text { mente grave }\end{array}$ & $\begin{array}{l}\text { Síntomas menos } \\
\text { alérgicos, asociado } \\
\text { con sinusitis }\end{array}$ & Eosinofilia, IL-5 & $\begin{array}{l}\text { Ac anti IL-5 y } \\
\text { antagonistas de los } \\
\text { receptores de los } \\
\text { cis-L. No responde a } \\
\text { corticosterioides }\end{array}$ \\
\hline $\begin{array}{l}\text { Asma inducida } \\
\text { por ejercicio }\end{array}$ & $\begin{array}{c}\text { Leve y posterior al } \\
\text { ejercicio }\end{array}$ & & $\begin{array}{l}\text { Activación de } \\
\text { mastocitos, citocinas } \\
\text { TH2 y cis-L }\end{array}$ & $\begin{array}{c}\text { Antagonistas de los } \\
\text { receptores de los cis-L, } \\
\text { beta-agonistas y Ac anti } \\
\text { IL-9 }\end{array}$ \\
\hline \multicolumn{5}{|l|}{ Fenotipo TH2-bajo } \\
\hline $\begin{array}{l}\text { Asma asociada } \\
\text { a obesidad }\end{array}$ & $\begin{array}{l}\text { Inicia en edad adulta } \\
\text { y adolescencia }\end{array}$ & $\begin{array}{l}\text { Principalmente } \\
\text { afecta mujeres, } \\
\text { muy sintomático } \\
\text { hiperrespuesta } \\
\text { aérea menos clara }\end{array}$ & $\begin{array}{c}\text { Pérdida de } \\
\text { marcadores } \mathrm{TH} 2 \text {, estrés } \\
\text { oxidativo }\end{array}$ & $\begin{array}{c}\text { Pérdida de peso, } \\
\text { antioxidantes, y } \\
\text { posiblemente a terapia } \\
\text { hormonal }\end{array}$ \\
\hline Asma neutrofílica & Inicia en edad adulta & Bajo FEV $_{1}$ & $\begin{array}{l}\text { Neutrofilia en esputo, } \\
\text { marcadores TH17, IL-8 }\end{array}$ & $\begin{array}{c}\text { Posiblemente a } \\
\text { antibióticos macrólidos }\end{array}$ \\
\hline
\end{tabular}

Fuente: (Bretón, Vargas, \& Miralles, 2015).

\section{Conclusiones.}

- El asma es na enfermedad que se desarrolla en edades tempranas, el conocimiento medico exhaustivo acerca de la fisiopatología de la enfermedad es la que va a desencadenar una nueva ola de tratamientos que puedan seguir atacando la enfermedad.

- La obesidad, el clima, el sexo, la genética y factores ambientales están asociados en el desarrollo del asma. 


\section{Fisiopatología de los problemas respiratorios en pacientes asmáticos}

Vol. 3, núm. 3 Esp., (2019)

Maria Monserrate Ganchozo Pincay; Ginger Alexandra Macías Corral; Marlon Jose Rivadeneira Rodriguez; José Andrés Sánchez Acebo

- El asma puede desarrollar diferentes tipos llegando a factores agudos y críticos que pueden desencadenar en la muerte de los pacientes.

- hay casos en que el asma aparece en las primeras etapas de la vida de un niño, pudiendo no presentarse más en la edad juvenil y adulta.

- El asmático es un paciente crónico difícil de manejar, que requiere de una terapia compleja; su manejo debe ser integral, y un punto básico es la educación del paciente y su familia.

- Existen tratamientos de medicina alternativa que, aunque no han tenido una validación científica, han sido exitosos en el control de los síntomas a largo plazo.

- El tratamiento del asma depende de cómo esta evolucione, ya que puede ser prolongado y hasta se pueden administrar diferentes combinaciones de medicamentos.

- Las pruebas de función pulmonar son de gran utilidad en el diagnóstico, evaluación de la gravedad de la obstrucción y en el seguimiento de la respuesta al tratamiento.

- Las pruebas de alergia son necesarias, principalmente para establecer si la exposición a alérgenos influye en el desarrollo del asma y con ello diseñar las medidas de control necesarias para evitar la exposición a alérgenos y la aparición de síntomas. 


\section{Fisiopatología de los problemas respiratorios en pacientes asmáticos}

Vol. 3, núm. 3 Esp., (2019)

Maria Monserrate Ganchozo Pincay; Ginger Alexandra Macías Corral; Marlon Jose Rivadeneira

Rodriguez; José Andrés Sánchez Acebo

\section{Bibliografía.}

Becerra, M. H. (2009). Fisiopatología del asma. NCT Neumología y Cirugía de Tórax, 68(52), $111-115$.

Bretón, N. R., Vargas, M., \& Miralles, E. (2015). Asma alérgica: mecanismos inmunológicos, fisiopatología y tratamientos actuales. Investigación y Ciencia, 23(65), 66-72.

Casanueva, C. O., \& Belinchón, J. (2012). Asma: educación sanitaria, autocontrol y medidas preventivas. PediatríaIntegral, 16(2), 141-148.

Durán, R. (2015). Fisiopatología del asma: una mirada actual. Revista Colombiana de Neumología, 27(3), 226-230.

García Merino, A., \& Mora Gandarillas, I. (2013). Diagnóstico del asma. Pediatría Atención Primaria, 15, 89-95.

González, R., \& Pérez, M. (2006). Aspectos fisiopatológicos. Neumología pediátrica, 1(2), 4953 .

Sienra-Monge, J. J., Río-Navarro, B., \& Baeza-Bacab, M, M. (1999). Asma. salud pública de méxico, 41, 64-70.

$$
\text { (c) } \frac{1}{\mathrm{BY}} \mathrm{NC} \text { (2) }
$$

RECONOCIMIENTO-NOCOMERCIAL-COMPARTIRIGUAL

CC BY-NC-SA

ESTA LICENCIA PERMITE A OTROS ENTREMEZCLAR, AJUSTAR Y CONSTRUIR A PARTIR DE SU OBRA CON FINES NO COMERCIALES, SIEMPRE Y CUANDO LE RECONOZCAN LA AUTORÍA Y SUS NUEVAS CREACIONES ESTÉN BAJO UNA LICENCIA CON LOS MISMOS TÉRMINOS. 